\title{
Database Dry Grasslands in the Nordic and Baltic Region
}

\author{
Jürgen Dengler \& Solvita Rūsiṇa
}

\begin{abstract}
The Database Dry Grasslands in the Nordic and Baltic Region (GIVD ID EU-00-002) is an initiative of the respective regional subgroup of the European Dry Grassland Group (EDGG). Its aim is to compile the available published and unpublished vegetation-plot data of dry grasslands and related syntaxa (mainly vegetation classes Koelerio-Corynephoretea, Festuco-Brometea, and Trifolio-Geranietea) from the study region (Denmark, Norway, Sweden, Finland, NW Russia, Belarus, Estonia, Latvia, Lithuania, $\mathrm{N}$ Poland, and NE Germany). The only requirement for inclusion is that the plot size is between 1 and $100 \mathrm{~m}^{2}$. The database is managed under Sort 4.0, but shall be transferred to TURBOVEG soon. Presently, 7,675 vegetation plots of the estimated 20,000 that exist within the scope are included. The strength of the database is that data from ten countries (no data available from Belarus yet) have been harmonised to one plant taxonomic view and that $70 \%$ of the plots have data on bryophytes and lichens alongside the vascular plants. The major aim of the originators of the database is to use it for consistent large-scale classifications. However, the data are also available for other research purposes upon specific agreement. Finally, EU-00-002 is one of the founding databases of the emerging European Vegetation Archive (EVA) as it contributes valuable data from a part of Europe where most countries do not have a national vegetation-plot database.
\end{abstract}

Keywords: bryophyte; dry grassland; European Dry Grassland Group (EDGG); European Vegetation Archive (EVA); FestucoBrometea; Koelerio-Corynephoretea; lichen; Trifolio-Geranietea; vascular plant; vegetation plot; vegetation classification.

\section{Database Dry Grasslands in the Nordic and Baltic Region}

Scope: All available relevés with plot sizes $1-100 \mathrm{~m}^{2}$ of dry grasslands and related communities (basically the phytosociological classes FestucoBrometea, Koelerio-Corynephoretea, and Trifolio-Geranietea sanguinel) from the study region (Denmark, Faroer, Iceland, Norway, Sweden, Finland, NW Russia, Belarus, Estonia, Latvia, Lithuania, N Poland, NE Germany)

Status: ongoing capture

Period: 1922-2009

Database manager(s): Jürgen Dengler (dengler@botanik.uni-hamburg.de); Solvita Rūsiņa (rusina@lu.Iv)

Owner: Working Group on Dry Grasslands in the Nordic and Baltic Region, a subgroup of the European Dry Grassland Group (EDGG)

Web address: http://www.biologie.uni-hamburg.de/bzf/syst/wg_dry_grasslands_nordic/wg_dgnb1_eng.htm

Availability: according to a specific agreement

Online upload: no

Online search: no

\section{Database format(s): Sort 4.0, to be transferred to TURBOVEG soon Export format(s): Excel}

Publication: Dengler, J., Rusina, S., Boch, S., Bruun, H. H., Diekmann, M., Dierßen, K., Dolnik, C., Dupré, C., Golub, V. B., Grytnes, J.-A., Helm, A., Ingerpuu, N., Löbel, S., Pärtel, M., Rašomavi?ius, V., Tyler, G., Znamenskiy, S. R., Zobel, M. (2006): Working group on dry grasslands in the Nordic and Baltic region - Outline of the project and first results for the class Festuco-Brometea. - Ann. Bot. N. S. 6: 1-28, Rome.

Plot type(s): normal plots

Plot-size range: $1-100 \mathrm{~m}^{2}$

Non-overlapping plots: 7,675

Estimate of existing plots: 20,000

Number of sources: 125

Completeness: $38 \%$

Total plot observations: 7,675

Valid taxa: 1,750

Countries: DE: 45.2\%; DK: 2.3\%; EE: 16.2\%; FI: 1.7\%; LT: 1.6\%; LV: 8.0\%; NO: 4.4\%; PL: 5.4\%; RU: 1.4\%; SE: 13.5\%

Forest: $0 \%$ - Non-forest: aquatic: $0 \%$; semi-aquatic: $0 \%$; arctic-alpine: $0 \%$; natural: $0 \%$; semi-natural: $100 \%$; anthropogenic: $0 \%$

Guilds: all vascular plants: $100 \%$; bryophytes (terricolous or aquatic): $71 \%$; lichens (terricolous or aquatic): $71 \%$; algae (terricolous or aquatic): $71 \%$; non-terricolous taxa (epiphytic, saxicolous, lignicolous): $4 \%$

Environmental data: altitude: $8 \%$; slope aspect: $62 \%$; slope inclination: $57 \%$; microrelief: $3 \%$; surface cover other than plants (open soil, litter, bare rock etc.): $13 \%$; soil pH: $17 \%$; other soil attributes: $19 \%$; land use categories: $3 \%$

Performance measure(s): cover: $100 \%$

Geographic localisation: small grid (not coarser than $10 \mathrm{~km}$ ): $75 \%$; political units or only on a coarser scale (>10 km): $25 \%$

Sampling periods: 1920-1929: 1.9\%; 1930-1939: 0.1\%; 1940-1949: 2.4\%; 1950-1959: 1.0\%; 1960-1969: 4.4\%; 1970-1979: 6.0\%; 1980-1989:

2.6\%; 1990-1999: 54.7\%; 2000-2009: $24.7 \%$

Information as of 2012-07-12; further details and future updates available from http://www.givd.info/ID/EU-00-002

Jürgen Dengler* (dengler@botanik.uni-hamburg.de)

Biodiversity, Evolution and Ecology of Plants (BEE), Biocentre Klein Flottbek and Botanical Garden, University of Hamburg,

Ohnhorststr. 18, 22609 Hamburg, GERMANY 
Solvita Rūsiņa (rusina@lu.lv)

Department of Physical Geography, Faculty of Geography and Earth Sciences, University of Latvia, 19 Raina blvd., 1586 Riga,

LATVIA

*Corresponding author 